\title{
COLECCION REPETIDA DE EMBRIONES Y TRANSFERENCIA INTERESPECIE EN ALPACAS Y LLAMAS DURANTE EPOCA NO REPRODUCTIVA
}

\author{
Repeated embryo collection and interspecies transfer in alpacas and llamas \\ during non-breeding season
}

Joel Pacheco', Signe Tollig², Amanda Wimblad Von Walter², Danilo Pezo', Victor Velez

http://dx.doi.org/10.18548/aspe/0002.1

'Estación IVITA Marangani-
Facultad de Medicina
Veterinaria - Universidad
Nacional Mayor de San Marcos
- Lima. Perú.
2 Facultad de Ciencias Agrícolas -
Universidad de Uppsala -
Suecia.
E-mail: jpachecoc@unmsm.edu.pe

pachecoc@unmsm.edu.pe

\section{RESUMEN}

Se realizó la evaluación del comportamiento sexual, la colecta y la transferencia de embriones interespecie en llamas y alpacas durante época no reproductiva, se utilizaron 10 alpacas y 10 llamas donadoras, 20 alpacas y 20 llamas receptoras, 5 alpacas y 5 llamas machos. Se evaluó el comportamiento sexual mediante la libido de los machos y la aceptación de las hembras al macho en presencia de un folículo dominante, se realizó la colecta de embriones de ovulación simple mediante la técnica no quirúrgica y se transfirió los embriones frescos directamente en el cuerno izquierdo. Se pudo observar que solo el $40 \%$ de las hembras alpaca acepto al macho y en todos los casos se tuvo que usar dos machos por monta, sin embargo en llamas todos los machos montaron en el primer intento y todas las hembras aceptaron la monta. Se colecto embriones en el 25 y $60 \%$ de los lavados en alpacas y llamas respectivamente, todos los embriones fueron grado 1 transferibles; la fertilidad de la transferencia embrionaria evaluada mediante ecografía a los 25 días fue de 100 y $41.6 \%$ para donadoras alpaca y llama respectivamente, sin embargo a la evaluación ecográfica a los 60 días la fertilidad fue de 50 y $25 \%$ para donadora alpaca y llama respectivamente. Se concluye que existe mayor estacionalidad reproductiva en las alpacas respecto a las llamas, todos los embriones colectados fueron grado 1, la fertilidad evaluada mediante ecografía desciende de los 25 días a los 60 días, evidenciándose mortalidad embrionaria, posiblemente por efecto de la época no reproductiva de ambas especies.

Palabras clave: transferencia embrionaria, alpaca, llama, época reproductiva, inter especie 


\section{ABSTRACT}

Sexual behavior evaluation was evaluated, collecting and interspecies embryo transfer inter species in llamas and alpacas during non-breeding season, 10 and 10 donor alpacas llamas, alpacas and 20 receiving 20 llamas, 5 alpacas and 5 llamas males were used. Sexual behavior by libido in males and acceptance of female to male in the presence of a dominant follicle was evaluated, the collection of embryos simple ovulation by non-surgical technique was performed and the fresh embryos are transferred directly into the horn left. It was observed that only $40 \%$ of alpaca accept the male and female in all cases had to use two males for mating, but all llama males mounted on the first attempt and accepted all females breeding. Embryos were collected at 25 and $60 \%$ of alpacas and llamas washes respectively, all were grade 1 embryos transferable; the embryo transfer fertility evaluated by ultrasound at 25 days was 100 and $41.6 \%$ respectively for donor alpaca and llama, however ultrasound evaluation at 60 days fertility was 50 and $25 \%$ respectively for donor alpaca and llama. We conclude that there is greater reproductive seasonality in alpaca regard to llamas, all were grade 1 embryos collected, fertility evaluated by ultrasound 25 days down to 60 days, demonstrating embryonic mortality, possibly due to the non-breeding season of both species.

Keywords: embryo transfer, alpaca, llama, reproductive season, inter species.

\section{INTRODUCCION.}

La transferencia embrionaria es una biotecnología que permite el mejoramiento genético al utilizar individuos superiores. Diversas investigaciones se están realizando en camélidos sudamericanos (CSA) para adecuar esta biotecnología a la fisiología reproductiva particular de estas especies (Huanca, 2005). En la zona alto andina, la temporada reproductiva de los CSA es estacional (Bravo, 1995), presentándose actividad reproductiva desde diciembre hasta abril, lo cual está relacionado a la época lluviosa y a la disponibilidad de pastos (Novoa, 1970).

En la actualidad, se viene implementando la técnica no quirúrgica en alpacas con ovulación simple, lográndose obtener $70 \%$ de efectividad de colección mediante esta técnica (Vaughan, 2012; Huanca et al., 2012). Se pueden realizar varios lavados sucesivos después de un periodo de descanso de las hembras donadoras, obteniendo $50 \%$ de embriones de calidad excelente y $25 \%$ de calidad bueno, lo que significa que el $75 \%$ de embriones recuperados serian transferibles (Pineda et al., 2012).
Por otra parte, la transferencia de embriones interespecie puede ser usada para lograr mejores tasas de gestación, transfiriendo embriones de alpaca en llamas receptoras, utilizando de esta manera la mayor habilidad materna de la llama, además de lograr tasas de preñez de 70 \% (Sumar et al., 2012), obteniéndose crías alpaca nacidas de receptora llama con mayores peso al nacimiento (Sumar et al., 2012; Huanca, et al., 2012). Pacheco et al. (2014) reportaron un $58.3 \%$ de recuperación de embriones en época reproductiva durante 4 lavados sucesivos en alpacas de ovulación simple. Existe evidencia de la posibilidad de realizar transferencias embrionarias viables en época no reproductiva en sudamerica (Von Baer et al., 2003).

El objetivo de este estudio fue evaluar el comportamiento sexual, la colección y transferencia inter especie de embriones de alpacas y llamas fuera de época reproductiva en la zona altoandina.

\section{MATERIALES Y MÉTODOS}

\section{Lugar de ejecución}

El trabajo fue ejecutado en la Estación Experimental IVITA Maranganí -Fundo La Raya, de la Facultad de Medicina Veterinaria de la Universidad Nacional Mayor de San Marcos, ubicada a 4200 m.s.n.m. el estudio se desarrolló durante los meses de septiembre y octubre.

\section{Animales}

Se usaron 10 alpacas y 10 llamas hembras como donadores y 20 alpacas y 20 llamas adultas como receptoras. Las hembras, con edades entre 4 y 8 años, fueron seleccionadas luego de la verificación de su capacidad reproductiva (cría en pie) y la factibilidad de realizar la palpación rectal. Los animales fueron alimentados en pasturas naturales, se utilizaron, 5 alpacas macho y 5 llamas macho, en edad reproductiva y buena condición corporal.

\section{Evaluación del comportamiento sexual}

Se evaluó el comportamiento sexual de la hembra mediante la observación de la aceptación o el rechazo a los machos, en presencia de folículo dominante determinada ecográficamente (Aloka SSD 225). El comportamiento sexual de los machos se evalúo observando la monta, si intentaba o no montar, o no presenta interés.

\section{Colección y transferencia de embriones}

Manejo de las hembras donadoras: se realizó la verificación de la presencia de folículo dominante mediante ecografía. Ante la presencia de un folículo dominante $\geq 7 \mathrm{~mm}$ Se realizó el empadre (día 0). El día 7 se realizó el lavado uterino mediante la técnica no quirúrgica. Posteriormente se aplicó un análogo de prostaglandina (Lutapros $₫$, Agrovet Market, cloprostenol, $0.025 \mathrm{mg}$, IM.) para destruir el cuerpo 
lúteo y asegurar el reinicio de la actividad ovárica. E día 15 se realizó el siguiente empadre y 7 días después se realizó el segundo lavado uterino. Se realizaron 2 lavados por donadora.

Receptoras: Fueron evaluadas ecográficamente para verificar la presencia de un folículo dominante. Se aplicó 8.2 ug de acetato de buserelina (Conceptase ${ }^{\circledR}$, Agrovet Market) como inductor de ovulación. La inducción de la ovulación se realizó el mismo día del lavado uterino de las donadoras, todas las transferencias fueron interespecie.

Se realizó el diagnóstico de preñez mediante ultrasonografía los días 25 y 60 post transferencia.

Para realizar el lavado uterino, se realizó una técnica no quirúrgica mediante el uso de una sonda de Foley y una jeringa descartable de $100 \mathrm{~mL}$, utilizando aproximadamente $100 \mathrm{~mL}$ de medio de lavado por cuerno. Se recuperó el fluido en un filtro EmCon. Para un manejo confortable de las hembras se administró

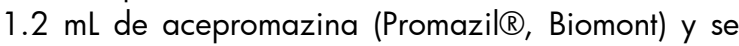
aplicó $1.5 \mathrm{~mL}$ de lidocaína al $2 \%$ via epidural (Otarvasq ${ }^{\circledR}$ S.A.C.).

La búsqueda de los embriones se realizó en lupa estereoscópica con platina temperada a $37^{\circ} \mathrm{C}$. Una vez ubicado el embrión, se colocó en medio de mantenimiento temperado, la evaluación se realizó usando la escala mencionada en el manual de la IETS (Stringfellow y Givens, 2011). Se utilizaron para transferencia solo los embriones categorías 1 y 2, los cuales fueron envasados en pajillas de $0.25 \mathrm{~mL}$.

La transferencia embrionaria se realizó por el método no quirúrgico, utilizando un aplicador de embriones con transfit lateral, depositando el embrión en el cuerno ipsilateral al folículo ovulatorio.

Se realizó la evaluación estadística mediante un diseño completo al azar y medidas de tendencia central, usando el programa SAS.

\section{RESULTADOS}

De las 10 alpacas donadoras, solo 5 y 3 de ellas aceptaron al macho en la $1^{\circ}$ y $2^{\circ}$ fecha de empadre respectivamente, en todos los casos se debió usar dos machos por copula puesto que no presentaron libido. De 10 llamas hembras, las 10 hembras aceptaron al macho en las dos fechas de empadre y no se necesitó recambio de machos, ambos grupos de hembras fueron evaluadas ecográficamente y se encontraban con ondas foliculares. Todos los embriones recuperados de alpacas y de llamas fueron $100 \%$ transferibles (todos grado 1). Los resultados de colección embrionaria en ambas especies se resumen en la tabla 1 y los resultados de fertilidad (diagnostico d gestación) se resumen en la tabla 1 .

Tabla 1. Recuperación repetida de embriones de alpacas y llamas y fertilidad post transferencia de embriones interespecie

\begin{tabular}{|c|c|c|c|c|c|c|c|c|c|}
\hline \multirow{2}{*}{$\begin{array}{c}\text { Especie } \\
\text { Donadora }\end{array}$} & \multicolumn{3}{|c|}{$1^{\circ}$ lavado } & \multicolumn{3}{|c|}{$2^{\circ}$ lavado } & & \multicolumn{2}{|c|}{ Diagnóstico preñez } \\
\hline & $\mathrm{n}$ & $\begin{array}{c}\# \\
\text { embriones }\end{array}$ & $\begin{array}{l}\% \\
\text { recup. }\end{array}$ & $\mathrm{n}$ & $\begin{array}{l}\# \\
\text { embriones }\end{array}$ & $\begin{array}{l}\% \\
\text { recup. }\end{array}$ & & $\begin{array}{c}\text { Ecografía } 21 \\
\text { días }\end{array}$ & $\begin{array}{c}\text { Ecografía } 60 \\
\text { días }\end{array}$ \\
\hline \multirow[t]{2}{*}{ LLama } & 10 & 6 & 60 & 10 & 6 & 60 & & & \\
\hline & & & & \multicolumn{3}{|c|}{ Transferencia interespecie } & Alpaca & $5 / 12(41.6 \%)$ & $3 / 12(25 \%)$ \\
\hline \multirow[t]{2}{*}{ Alpaca } & 5 & 0 & 0 & 3 & 2 & 67 & & & \\
\hline & & & & \multicolumn{3}{|c|}{ Transferencia interespecie } & Llama & $2 / 2(100 \%)$ & $1 / 2(50.0 \%)$ \\
\hline
\end{tabular}

\section{DISCUSION}

Solo el $40 \%$ de las alpacas sometidas a empadre fuera de época reproductiva presentaron comportamiento sexual positivo, a pesar de estar en fase folicular determinada mediante ecografía, lo cual concuerda con reportes anteriores sobre la estacionalidad reproductiva de los camélidos (Novoa, 1970; Bravo, 1995; Pollard et al., 1995), sin embargo este comportamiento solo fue observado en las alpacas, puesto que en las llamas, el $100 \%$ presento comportamiento sexual positivo, siendo esto último, posiblemente una característica propia de la especie.
La eficiencia de recuperación de embriones fue de 25 $\%$ en alpacas donadoras simples, este resultado es inferior al reporte de Pineda et al. (2012), quienes indican $61.54 \%$, dicho reporte fue realizado en condiciones medioambientales muy diferentes al del presente estudio, sin embargo también es inferior al reporte de Pacheco et al. (2014) quienes indican 58.3 $\%$ de recuperación de embriones de alpacas de ovulación simple en las mismas condiciones de altura, pero en época reproductiva, por lo que podríamos asumir que estos bajos resultados estarían influenciados por la época del año y las condiciones alimenticias deficientes y diferentes a las del anterior 
estudio. La recuperación de embriones de llamas de ovulación simple fuera de época reproductiva fue del $60 \%$, lo cual se repite en ambos lavados sucesivos, lo cual es similar a lo reportado por Huanca et al. (2014), quienes indican que la eficiencia de recuperación de embriones de llama de ovulación simple es de 58.9\%.

El $100 \%$ de los embriones colectados en alpacas y llamas fue de muy buena calidad, grado 1 en todos los casos, lo cual es superior a los reportes previos que indican alrededor del $70 \%$ de embriones transferibles en lavados uterinos de animales de ovulación simple (Pineda et al., 2012; Pacheco et al., 2014; Huanca et al., 2014).

La fertilidad de la transferencia embrionaria en alpacas y llamas fue alta para la primera evaluación ecográfica a los 21 días, así tenemos en receptoras llamas $100 \%(2 / 2)$ y $41.6 \%(5 / 12)$ en receptoras alpacas, siendo superior la habilidad materna de la llama con respecto a la alpaca, tal como lo indican Huanca et al. (2014), sin embargo la fertilidad disminuye cuando se realiza la segunda ecografía a los 60 días, teniendo $50 \%$ en receptoras llamas y 25 $\%$ en receptoras alpacas, posiblemente debido a la mortalidad embrionaria ya antes descrita en camélidos sudamericanos (Bravo et al. 2010). La fertilidad para alpaca y llama (50 y $25 \%$ ) es similar a lo descrito en transferencia de embriones interespecie en puna seca durante época reproductiva (Huanca et al. 2014), quienes indican 53.85 y $27.58 \%$ para donadoras alpaca y llama respectivamente.

\section{CONCLUSIONES}

Existe menor actividad reproductiva en alpacas, mientras que las llamas tanto machos como hembras tuvieron una actividad reproductiva normal durante época no reproductiva; la colección embrionaria y la fertilidad fueron similares a otros reportes realizados durante época reproductiva. La capacidad reproductiva de la llama receptora en el presente trabajo fue superior a la de la alpaca receptora, sin embargo se evidencia alta mortalidad embrionaria, posiblemente debido a las malas condiciones medioambientales en época no reproductiva.

\section{REFERENCIAS}

- Bravo W. Physiology of Reproduction in the Female Alpaca, Rev. Camelids N ${ }^{\circ}$ 7; Ed Pos Graduate Foundation in Veterinary Science, Sydney Australia. 1995.

- Bravo W, Díaz D, Alarcón V, Ordoñez C. Effect of the reproductive state of female alpacas on embryonic mortality rate. Am. J. Vet. Res. 2010; 71(9): 1096-1099.
- Huanca WL. Aplicación de biotecnologías reproductivas en especies domésticas y silvestres de camélidos sudamericanos. Agrociencia. 2005; 9(1-2): 505-509.

- Huanca T, Mamani RH, Cárdenas O, Gonzales ML, Sapana R. Evaluación del peso al nacimiento, destete, al año de edad y curva de crecimiento de alpacas y llamas cría nacidas por transferencia de embriones interespecies. Spermova, 2012; 2(1): 44-46.

- Huanca T, Gonzales ML, Mamani-Cato GH, Cárdenas O, Sapana R, Naveros M. Evaluación de la recuperación de embriones de alpacas y llamas donadoras simples y superestimuladas. Resúmenes del XXXVII Reunión Científica Anual de la Asociación Peruana de Producción Animal. APPA Abancay, Perú. 2014.

- Novoa C. Reproduction in Camelidae. A review. J. Reprod. Fertil. 1970. 22, 3-20.

- Pacheco JI, Pezo SD, Velez VM. Eficiencia de la colección y la calidad de embriones de alpaca obtenidos a partir de ovulaciones simples sucesivas. Spermova 2014; 4(2):159-161.

- Pineda J, Pozo A, Huanca T, Naveros ML. Recuperación sucesiva de embriones y retorno folicular post lavado en alpacas (Vicugna pacos) huacaya donadoras naturales. Spermova 2012; 2(1): 53-54

- Pollard JC, Littlejhon RC, Moore GH. Seasonal and others factors affecting the sexual factors in alpacas. Anim Reprod. Sci. 1995; 27(3-4): 349356.

- Stringfellow D, Givens D. Manual de la Sociedad Internacional de Transferencia de embriones. Cuarta Edicion. International Embryo transfer Society. Illinois. USA. 2011.

- Sumar J, Arellano P, Montenegro V, Londoñe P, Picha Y, Rodriguez C, Sanchez D, Torres R. Reciprocal embryo transfer in alpacas and llamas. Programme and extended abstracts. ICAR 2012. Satellite meeting on camelid reproduction. Vancouver-Canada. 2012.

- Vaughan JL. Embryo transfer in alpacas. Programme and extended abstracts. ICAR 2012. Satellite meeting on camelid reproduction. Vancouver-Canada. 2012.

- Von Baer A, Von Baer L, Donoso M, Poblete P, Miranda H, Del Campo M. Efecto de diferentes parámetros en un programa de transferencia de embriones en llamas (Lama glama) y alpacas (Lama pacos). Resúmenes del III Congreso Mundial sobre Camélidos. Potosi, Bolivia. 2003. 